\title{
Oser le corps
}

\section{Alex CORMANSKI}

La pluridisciplinarité réserve parfois la surprise de l'entrecroisement des champs qu'elle explore, par exemple celui de la géologie et de la transgression. Il n'est pas interdit de penser que nombre d'hommes et de femmes de science, même versés en la matière, ont découvert qu'au début du vingtième siècle, la première, la science de la terre, celle qui traite des couches du sol, des strates, de l'assiette terrestre, s'était appropriée la seconde, la transgression, nous révélant son sens mélioratif, sa bonne nature en quelque sorte l'envahissement des terres par la mer suite à un affaissement -, ce qui équivaudrait somme toute à l'action de combler un vide, ce dont la nature a horreur comme nous le savons tous.

La transgression amènerait donc réparation pour recomposer, pour réinvestir des champs perdus, ou délaissés, ou peut-être un terrain perdu, non reconnu.

Dans un autre domaine, celui des sciences humaines, elle garde le sens de franchissement, de dépassement des limites, des règles, outre-passement des interdits, des tabous, dénominateur commun à toutes les cultures et civilisations. Reconquête de terrain d'un côté, dépassement des règles de l'autre, les deux acceptions seraient-elles complémentaires ? Est-ce à dire que le terrain à reprendre était du domaine de l'interdit, raison pour laquelle il n'était pas exploré ?

Poser la question de la transgression en didactique des langues et des cultures ne saurait surprendre tant celle de son pendant, la norme, se pose elle aussi, de prime abord sur le plan strictement linguistique (phonétique, morphologie, sémantique). On se doit d'y faire référence quasiment dans toute situation d'enseignement, encore plus en milieu institué, et tout enseignant en principe s'y conforme. Mais il ne peut non plus faire abstraction de ce qui vient bousculer la même norme, des irrégularités néologisantes dans l'usage de la langue - il s'en délectera parfois même et les singularisera dans son enseignement d'autant mieux qu'elles finiront assez vite par être «normalisées » comme le souligne Berthille Pallaud. Autrement dit, point d'expulsions à craindre, la régularisation des nouveaux intrus ne sera que simple épreuve de passage.

Par ailleurs, si la transgression prend le sens de « co-construction entre une personne et des situations » comme le définit Philippe Meirieu, et non plus seulement celui de contrevenir à une règle, elle a alors tout d'une dynamique de réappropriation de terrain abandonné ou peut-être non reconnu, où le goût de l'aventure a toutes les chances d'être 
récompensé, étant surtout, avant d'être une invitation à dépasser ou encore outrepasser, une invitation à re-connaître. Ce terrain à re-connaître, c'est celui du corps en commençant par le constat, longtemps de mise, que la langue d'enseignement a trop souvent été « décorporalisée ».

Le corps, matrice incontournable du processus d'énonciation, aussi bien du point de vue des marques prosodiques du discours que de celui de la production linguistique à partir d'interactions entre locuteurs, liant verbal et non-verbal (cf. le « ligament grammatical » dont parlait Charles Bally, disciple de Saussure), base de l'expressivité, a longtemps été étranger, non incorporé, au processus d'apprentissage de la langue.

Recorporaliser la langue, c'est partir en terrain de re-connaissance, c'est réinvestir le champ perdu du signe, pourtant tellement empreint de signifiance, que le corps orchestre dans la construction du discours par le jeu des interactions.

Oser le corps, «cette structure anthropologique, cause et effet d'une intensification de l'activité sociale »(Maffesoli) pour le réhabiliter, c'est oser le rapport à l'autre dans la construction de l'interaction avec cet autre co-apprenant impliqué dans un processus d'apprentissage partagé, c'est affronter la question de la gestion des craintes et des angoisses dans l'inter compréhension pour pouvoir énoncer, opération d'autant plus complexe à gérer quand l'identité culturelle des apprenants est diverse (contexte d'apprentissage en milieu hétéroglotte avec par exemple une classe composée d'apprenants japonais, latino-américains ou occidentaux en France). C'est aussi connaître le plaisir des voix communicantes, des constructions qui s'équilibrent entre le faire et le dire comme cela se fait « naturellement » dans la communication courante entre locuteurs natifs dans une intersynchronie bien partagée.

$\mathrm{Si}$, apprendre à communiquer en langue étrangère, c'est aussi apprendre à savoir interagir socialement en milieu naturel (le co-agir fortement mis en avant par le CECRL), la classe-langue, simulacre de la scène du quotidien - le fameux «si magique » que travaille le comédien (Stanislavski) -, peut être le lieu propice où mettre en exercice ce rapport à l'autre grâce à la pratique du jeu - constitutif de l'apprentissage comme dit Lev Vygotski - et tel qu'il s'élabore par la pratique des techniques dramatiques. On peut définir celles-ci comme l'ensemble de ce qui est mis en œuvre pour travailler la voix, instrument premier de l'énonciation (prononciation, intonation, articulation, rythme), la gestuelle, la mimique, la posture, à l'instar de ce que fait un comédien de théâtre à l'entrainement si l'on peut dire. Dans le contexte de la didactique, ces techniques visent dans un premier temps à «recorporaliser» la langue d'enseignement pour favoriser les interactions par le jeu 
dramatique. Jeu qui au demeurant met en scène un certain nombre de « je » qui, comme le souligne Jan Assmann dans La mémoire culturelle se développent toujours de l'extérieur vers l'intérieur chez l'individu du fait que ce dernier participe aux structures d'interaction et de communication de son groupe et s'identifie à l'image que le groupe a de soi, l'identité étant un phénomène social, étant « sociogène ».

L'identité collective, le «nous » n'existe pas en dehors des individus qui la portent et la constituent. Elle doit être un objet de connaissance et de consciences individuelles.

Assman définit deux types d'identité :

- une identité individuelle comme une image élaborée et entretenue, dans la conscience de l'individu, des traits particuliers qui le distinguent de tous les autres (les «autres significatifs »). Elle est la conscience, développée grâce au fil conducteur qu'est le corps, de sa spécificité irréductible, de son caractère unique et irremplaçable. Elle est liée à la contingence d'une vie avec ses « dates angulaires » de naissance et de mort, au fait que l'existence et ses besoins fondamentaux s'incarnent dans un corps.

- une identité personnelle qui est la somme de tous les rôles, qualités et compétences qu'impose à l'individu son appartenance à des constellations spécifiques de la structure sociale. Elle est liée à la reconnaissance sociale de l'individu responsable de ses actes. Toutes deux sont « sociogènes » et déterminées culturellement. Le « je » est toujours une construction sociale et, à ce titre, une identité culturelle.

Comment ce «je »s'énonce-t-il dans la langue étrangère, en situation d'apprentissage d'une langue étrangère ? Qu'est-ce même qu'énoncer dans une langue étrangère ? Comment fonctionne le corps dans le transfert de corporéité, dans l'appropriation de la tonalité et de la mélodie vocale de la langue cible, qui peut être marque de reconnaissance intégrative dans le groupe social, ou bien encore dans la variable comportementale (attitude, posture, voix) très marquée au niveau du corps dans certaines cultures comme en japonais par exemple plus que dans d'autres. Ainsi, comment un locuteur japonais, habitué à accomplir l'acte social élémentaire de saluer dans son pays, qui passe par une inclinaison du corps variable selon le statut de son interlocuteur, devra-t-il s'adapter pour réaliser le même acte en France ? L'interface culturelle, qui consisterait à voir un locuteur natif adopter et maîtriser la norme comportementale en vigueur dans son pays quand il y séjourne et celle du pays cible quand il s’y rend, n'est pas impossible à acquérir, mais elle reste assez rare à vérifier.

L'exemple suivant peut toutefois servir d'illustration pertinente et appuyer ce dernier point. La scène a lieu à l'aéroport de Milan. Une grosse voiture s'arrête près de la porte du hall d'entrée. Un homme, de type européen, en sort vite pour ouvrir la portière au passager 
assis à l'arrière, visiblement un homme d'affaires important, de type asiatique, en apparence japonais. Il s'incline devant lui, lui tend sa serviette des deux mains, s'incline de nouveau de façon légèrement plus prononcée, et maintient sa posture tout en s'exprimant en langue nipponne. L'homme d'affaires prend la serviette, dit quelques mots à voix basse, puis se dirige vers la salle d'embarquement. L'homme qui avait tenu son rôle d'employé très attentionné, se redresse, puis sort son téléphone portable de sa poche et se met à parler dans sa langue maternelle selon un mode d'expression verbale et une gestuelle très identifiable comme italien. La variable comportementale (attitude, posture, gestuelle, voix) d'une langue et d'une culture étrangères, dans une situation précise, ici professionnelle, est dans ce cas précis tout à fait maîtrisée et ceci dans un contexte d'apprentissage, même si le référent culturel cible est à priori assez éloigné de la culture d'origine. Observer la même chose avec des apprenants japonais en italien reste peut-être à montrer ou démontrer. Toujours est-il que la capacité du corps à se reformater pour s'acclimater, pour être en phase avec un nouvel environnement reste de l'ordre du réalisable.

Oser le corps, c'est aussi oser le rapport à soi. Enoncer dans une langue étrangère, c'est explorer une nouvelle tonalité, une nouvelle résonance, c'est chercher à s'approprier un nouveau mécanisme opératoire de l'énonciation, qui passera très certainement par une adaptation de l'appareil phonatoire, pour être capable d'articuler, de prononcer juste musicalement parlant (« to be attuned » en anglais). «Sonner» comme un natif ou presque, (le stress tonique de prononciation pour ce qui est de l'anglais par exemple), est souvent une tâche programmée et un critère d'évaluation de la compétence orale dans le contexte d'un apprentissage des langues à visée professionnelle. Ne s'agit-il pas d'une reconnaissance de sa parole dans une autre langue, qui implique de passer d'un état de handicap à celui de maîtrise (ne plus sonner étrange dans la langue cible), pour éviter de se sentir discriminé dans son identité, une épreuve hautement «sociogène » extrêmement engageante.

Un professeur d'anglais dans une grande université parisienne faisait un jour valoir en privé que les Français semblent mettre un frein à dessein à s'exprimer dans la langue de Shakespeare de façon aussi proche que possible des Britanniques car ils jugeraient alors leur modalité tonale comme trop artificielle, quasi dénaturée au risque de les faire passer pour ce qu'ils ne voudraient pas être.

A l'inverse, des Français s'exprimant en anglais outre-Atlantique se complaisent parfois, d'après un locuteur natif, à adopter un certain style qu'il s'amusait à qualifier d'accent « cow-boy » dans leur façon de traîner sur certaines voyelles. 
Si cela relève de l'anecdote, il n'est pas vain cependant d'observer la concomitance entre voix et corps dans le phrasé, comme si la mécanique corporelle ne pouvait qu'être au diapason dans la production des sons. On fait valoir également que le corps dit «loose » (détendu) des Américains va de pair avec leur phrasé, les deux étant non seulement en conformité mais aussi en consonorité.

Faut-il alors parler de transgression obligée des représentations culturelles que l'on a vis-à-vis de la langue et de la culture cible, de transgression de sa propre représentation pour ouvrir une autre voix/voie?

Le travail sur le corps par le corps n'est-il pas au cœur de cette « dimension constructive de la notion de transgression linguistique et culturelle que nous souhaitons privilégier ici, pour tâcher de lui rendre la richesse de sa valeur progressive, celle qui peut nous faire aller de l'avant et pour cela compléter le concept de limite. Selon cette optique, on s'interrogera alors sur un nouvel espace à trouver ou à créer dans une tension entre transgression de règles et désir de s'émanciper » (Meirieu).

Faire travailler l'apprenant sur le sens par la voix, dans une situation d'enseignement/apprentissage, n'est-ce pas entrer dans la langue de façon quasi clandestine (parce que non admise en général, en fait faussement illégale) en faisant croire (non pas au sens de duperie mais plutôt d'intime conviction) à l'apprenant que la langue est une affaire interne, qu'elle s'apprend de l'intérieur de soi. «C'est la voix qui donne la signification, non les termes du langage » dit David Le Breton dans Eclats de voix. «Elle signifie par son intonation, son rythme, elle est un message susceptible de troubler ou de déborder les significations de la parole tenue » ajoute-t-il.

Trouver le sens par la voix implique aussi la contre-voix, le silence, qui fait partie de l'économie de la parole. «Ne plus écouter celle-ci, mais seulement la qualité de sa formulation, de ses vibrations sonores, affectives, ses singularités » en fait partie dit encore Le Breton.

Bref, il n'y a pas de voix sans corps. Faire jouer les sonorités, créer des dialogues par le jeu de ces sonorités révèlent l'outil premier, l'articulateur essentiel de la prosodie de chacun qu'est le corps.

Mais mobiliser le corps, premier habitus culturel de l'apprenant parce qu'émanation et représentation de sa propre culture, peut-il se faire de façon identique au regard de la diversité culturelle ? Le corps peut-il/doit-il être pensé comme nécessairement altéritaire dans le cadre de l'apprentissage/enseignement des langues ou bien reste-t-il réfractaire à tout transfert de compétence identitaire, vivant du coup l'altérité comme «antipathique au «bon sens » 
(Barthes) ? Le transfert de corporéité peut-il opérer aisément ? Les résistances ne manqueront pas.

Certains affirment qu'un même individu parlant plusieurs langues dispose de plusieurs voix liées aux sons et à la mélodie qu'il imprime à chacune ; son corps s'ajusterait à la langue parlée. Le Breton cite l'exemple des Indiens Kutenaïs du sud-ouest canadien qui changent l'expression de leur voix, modifient leur gestuelle dès lors qu'ils quittent leur langue traditionnelle au profit de l'anglais. Il cite aussi Birdwhistell qui donne l'exemple de l'ancien maire de New York, la Guardia, qui était capable de parler couramment italien, américain et même yiddish et changeait de voix et de gestes selon la langue utilisée.

Sont-ce là des stratégies discursives diverses révélant le caractère complexe de l'identité ?

Freud cite l'exemple de cette baronne autrichienne, sur le point d'accoucher, qui voit le médecin accoucheur en présence du mari de celle-ci différer son intervention selon qu'il entend les cris de la patiente d'abord en français, puis en allemand et finalement décider d'intervenir quand celle-ci s'exprime en yiddish. La vérité se manifeste-t-elle seulement quand le verbe fait chair?

Nous n'entendons pas notre voix comme l'entendent les autres, nous ne l'entendons que filtrée par l'opacité de la chair selon Le Breton. Dès lors comment trouver sa voix dans la langue étrangère ? Celle-ci ne pourra-t-elle jamais être qu'étrangère ? Si « changer de voix est une équivalence du déguisement, une manière de donner le change en n'étant plus reconnaissable » comme le propose le même Le Breton, provoquant alors « un désir de jeu pour se libérer des allégeances au sérieux de la vie quotidienne », le terrain est grand ouvert au travail de façonnage de la voix comme on peut le faire dans le jeu dramatique dans la classe de langue avec les apprenants à condition d'en assumer cette nécessaire dimension.

Par ce biais, l'apprenant joue avec son corps, loin de la faute qui le restreint toujours, il l'oublie, ou tout simplement apprend à faire avec. Serait-ce là une bonne école pour transgresser ?...

Comme le dit encore un expérimentateur averti de la langue, qui a « adhéré » comme il dit au français, l'écrivain japonais Akira Mizubayashi, qui se dit prêt à « exposer sa voix nue, à dévoiler par sa voix sa manière absolument singulière d'exister, à vivre une dénudation », la parole vraie, authentique, «fautive » peut-être, mais lourde de sens et infiniment persuasive dans une situation d'énonciation liée à la mort ou à la naissance, est une parole articulée à mille lieues du souci de la correction ». A l'instar de la baronne de Freud, Mizubayashi cite l'exemple d'une femme française accouchant au pays du soleil 
levant qui «sonnait» si vrai dans son annonce à la vie, même si on pouvait noter quelque « dis-correction » (néologisme assumé) dans sa parole. Pareil propos tenu par un auteur nippon n'est pas sans intérêt.

En effet, la signification du concept de compétence orale dans son sens large et l'acceptation de ce qu'il recouvre selon les différents publics apprenants venus d'horizons culturels différents ne correspondent pas toujours aux mêmes représentations et usages que l'on peut avoir selon le monde auquel on appartient, en l'occurrence entre le monde occidental et le monde oriental. La question de l'interculturel, comme de l'intraculturel, est d'emblée posée, comme celle, déterminante, de la représentation, d'où l'enjeu sur le plan didactique et pédagogique.

La compétence orale dans la culture japonaise n'est pas assimilable à ce qu'elle peut être dans la culture française. Prendre la parole par exemple n'est pas un acte encouragé dans le système éducatif japonais quand il est vu au contraire comme un signe d'affirmation de soi, de sa volonté de participer en Occident. C'est encore Mizubayashi qui dit que « tout en parlant français, il conserve en lui comme une cicatrice ineffaçable, l'écho et l'empreinte de l'être-ensemble japonais ».

Dans la même veine, il n'est pas rare d'entendre des collègues originaires de cette même zone identitaire culturelle occidentale mettre en doute la faisabilité d'une approche de la langue par les techniques dramatiques, centrée sur le corps de l'apprenant, avec le public asiatique. Là encore, la transgression des idées reçues ne devrait-elle pas être inscrite dans tout curriculum ? Car, outre le fait que telle affirmation révèle de la part de ceux qui l'énoncent une certaine arrogance dans leur manière d'ordonner le monde de façon plutôt monolithique - comme s'il n'y avait qu'un seul et même public asiatique dans cette partie de l'hémisphère où la diversité linguistique, culturelle, ethnique est constitutive de la norme de chacun des pays qui la compose -, les mêmes faiseurs d'ordre catégoriel semblent avoir un préjugé tenace quant au mode d'expressivité corporelle de ces mêmes publics qu'ils assimilent à un quasi handicap. C'est presque un sans corps pointé. Or, le corps asiatique, dans ses nombreuses variantes culturelles, est extrêmement présent dans la codification des actes sociaux, le répertoire de sa fonctionnalité couvre un champ peut-être plus étendu que dans le monde occidental.

Ce propos a pu être vérifié lors d'ateliers de pratiques de classe auprès de professeurs dans le cadre de formations pédagogiques dans le sud-est asiatique (Cambodge 2010, 2011, 2012, Laos 2011, Vietnam 2011, Thaïlande 2010, 2012, Malaisie 2010, 2012), et aussi directement auprès d'étudiants du secondaire et du supérieur (Thaïlande), théâtre de champs 
d'expérimentation de l'enseignement/apprentissage du français par lesdites techniques dramatiques et le résultat fut des plus probants dans l'acceptation de l'approche et surtout dans les résultats obtenus : fluidité, spontanéité, créativité de la part des apprenants comme des enseignants.

Pour commencer, un travail sur l'énonciation, travail sur la voix par exemple, à partir d'exercices de respiration abdominale en interaction par groupes de deux, proposé dans chacun des pays cités, n'a jamais suscité la moindre résistance de la part des publics concernés conscients de la nécessité d'émettre le son depuis cette partie du corps, comme en atteste la pratique corporelle dans le domaine du théâtre et de la danse au plan local.

Lors de la mise en place de jeux interactifs, ensuite, la participation spontanée de chacun a été immédiate, le déroulé de l'interaction fourni et équilibré, l'auto-synchronie de chaque locuteur (équilibre entre le non-verbal et le verbal dans le discours, marques prosodiques du discours, gestuelle appropriée, confiance en soi dans la prise de parole) parfaitement ajustée. Si cette gestuelle n'avait pas un caractère expressionniste, en référence à ce qui peut être observé dans le monde occidental, elle n'en était pas moins très authentique et adéquate à la situation définie dans son expressivité. Et c'est bien là l'unique façon d'évaluer ce qui est énoncé, produit dans la combinatoire verbal/non-verbale, comportementale dans quelque partie du monde que ce soit. Peu importe dans quelle amplitude gestuelle, à quel degré sonore, dès l'instant que puisse être reconnue une véritable authenticité dans ce qui est acté en fonction de la situation donnée. On pourra éventuellement y reconnaître une caractéristique culturelle particulière, une touche identifiable, comme appartenant davantage à une partie du monde qu'à une autre, mais le critère d'évaluation de l'inter-synchronie entre les inter-actants devra répondre à des normes identiques. La question de la référence à un quelconque modèle ne se pose pas parce qu'elle est en fait difficilement posable.

Existe-t-il en effet une façon standard d'exprimer un quelconque sentiment modélisable et modélisé dans le cadre de l'apprentissage d'une langue étrangère ? Il est peut-être plus approprié de parler d'une économie des marqueurs de l'énonciation qui doivent fonctionner comme un ensemble équilibré et ajusté, ceci quel que soit l'environnement culturel.

Entre méconnaissance d'une diversité culturelle authentique et représentation hégémonique de celle-ci, la transgression peut certainement combler du terrain.

Le corps est, on le voit, sujet de discours, de discorde, de crainte, de souffrance et de plaisir, de méconnaissance et de reconnaissance. Il est le chantier de construction du dire où 
se forge la parole dans le vent des sonorités étranges de l'autre langue avec laquelle on se familiarise jusqu'à ce qu'elle ne soit plus étrangère. La manière de parler a un caractère préverbal malgré le fait qu'elle est parfaitement intégrée à l'acte verbal proprement dit comme dit Ivan Fonagy. Inutile donc de donner de la langue avant d'avoir émis le souffle. On ne fait que revenir au corps.

Passer d'une langue à une autre, c'est toujours jouer du corps, ce qui implique à l'évidence de bousculer son habitus corporel, c'est-à-dire culturel. Se doit-on d'être en état de transgression permanent?

\section{Bibliographie}

Assman, Jan. La mémoire culturelle. Ecriture, souvenir et imaginaire politique dans les civilisations antiques. Trad. de l'allemand Diane Meur. Paris: Aubier, 2010.

CECRL. Conseil de l'Europe. Paris: Didier, 2001.

Cormanski, Alex. Techniques dramatiques : activités d'expression orale. Paris: Hachette, coll. «Pratiques de classe », 2005.

Cuq, Jean-Pierre, dir. "Hétéroglotte». Dictionnaire de didactique du français langue étrangère et seconde. Paris : Asdifle - CLE International, 2003.

Fonagy, Ivan, La vive voix, essai de psycho-phonétique. Préface Roman Jakobson. Paris : Payot, 1991.

Le Breton, David. Éclats de voix. Une anthropologie des voix. Paris: Métailié, coll. «Traversées », 2011.

Maffesoli, Michel. Une symbolique du corps. Le corps tabou. Internationale de l'imaginaire 8, Paris : Actes Sud, 1998.

Meirieu, Philippe. «Apprentissage et transgression». Communication. Entretiens Nathan, 2003.

Mizubayashi, Akira. Une langue venue d'ailleurs. Paris: Gallimard, 2011.

Pallaud, Berthille. «Les disfluences et quelques autres libertés de langage : de l'émancipation à la progression ». Communication. Colloque «La transgression : de l'émancipation à la progression », UCO, Angers, 2013.

Stanislavski, Constantin. La formation de l'acteur. Paris: Payot, coll. «Petite bibliothèque », 2001.

Vygotski, Lev. Pensée et langage. Paris : La Dispute, 2003. 